\title{
Downregulated expression of CFHL1 is associated with unfavorable prognosis in postoperative patients with hepatocellular carcinoma
}

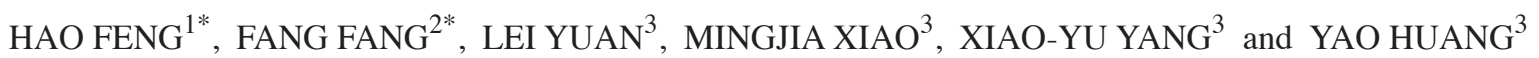 \\ ${ }^{1}$ Department of Gynecology and Obstetrics, Obstetrics and Gynecology Hospital of Fudan University, Shanghai 200090; \\ ${ }^{2}$ Department of Radiotherapy, Navy Medical University, Shanghai 200433; ${ }^{3}$ Department of Biliary Tract Surgery, \\ Eastern Hepatobiliary Surgery Hospital, Navy Military Medical University, Shanghai 200438, P.R. China
}

Received June 7, 2018; Accepted February 15, 2019

DOI: $10.3892 /$ etm.2019.7455

\begin{abstract}
Complement factor H-related protein 1 (CFHL1) was recently reported to be a potential biomarker in several types of cancer. CFHL1, however, has not been found to be of prognostic value in hepatocellular carcinoma (HCC) to date. In the present study, the expression levels of CFHL1 were evaluated in 8 pairs fresh frozen tissue samples using western blotting. Furthermore, the expression level of CFHL1 was evaluated in 76 pairs of formalin-fixed, paraffin-embedded (FFPE) HCC and peritumoral tissues (expression pattern cohort), and 278 FFPE HCC tissues (prognostic cohort) using tissue microarray-based immunohistochemistry. The Kaplan-Meier method, Cox regression proportional hazard model and receiver operating characteristic curve analysis were used to evaluate prognostic factors. The expression level of CFHL1 was reduced in HCC tissues in $67.1 \%$ (51/76) of the cases compared with the corresponding peritumoral tissues. Survival analyses indicated that patients with HCC with low CFHL1 expression had a worse time-to-recurrence (TTR) and overall survival (OS) compared with those with high CFHL1 expression in the prognostic cohort $(\mathrm{P}=0.002$ for $\mathrm{OS}$ and $\mathrm{P}=0.017$ for TTR). Both univariate and multivariate analyses indicated that CHFL1 was an independent prognostic factor for TTR and $\mathrm{OS}(\mathrm{P}=0.017$ and $\mathrm{P}=0.002$, respectively). In addition, The Cancer Genome Atlas (TCGA) and Human
\end{abstract}

Correspondence to: Dr Xiao-Yu Yang or Dr Yao Huang, Department of Biliary Tract Surgery, Eastern Hepatobiliary Surgery Hospital, Navy Military Medical University, 225 Changhai Road, Shanghai 200438, P.R. China

E-mail: ehbh_yangxy@163.com

E-mail: huangyao@vip.163.com

*Contributed equally

Key words: complement factor H-related protein 1, hepatocellular carcinoma, liver resection, prognosis
Protein Atlas were used for further validation. Furthermore, a prognostic model included tumor size, tumor number, liver cirrhosis and CFHL1 expression was evaluated. The results of the present study demonstrated that CFHL1 was downregulated in $\mathrm{HCC}$ and its level was associated with patient prognosis; therefore, CFHL1 is a potential prognostic marker for HCC.

\section{Introduction}

Liver resection is considered to be the most effective therapy for patients with hepatocellular carcinoma (HCC) to date. However, due to the high incidence of tumor recurrence and metastasis, the overall prognosis of HCC remains unsatisfactory (1). It has been reported that $>70 \%$ of patients with HCC develop recurrence within 5 years after surgery (2). The mechanism underlying recurrence and metastasis of HCC is complex and remains unclear. Therefore, novel molecular prognostic biomarkers of $\mathrm{HCC}$ are required to improve the survival of patients with HCC (3). Complement factor H-related protein 1 (CFHL1), a member of the complement factor $\mathrm{H}(\mathrm{CFH})$ family, was demonstrated to inhibit the activity of C5 convertase, as well as the assembly and membrane insertion of the terminal complement components $(4,5)$. It was also reported that human CFHL1, had a function and structure similar to human CFH (6). Several studies reported that CFHR is associated with bladder cancer and may be used as a quantitative urinary tumor marker in selected patients (7,8). In 2011, Yang et al (9) revealed that $\mathrm{CFH}$ exhibited decreased mRNA expression and increased $\mathrm{CpG}$ site methylation in surgically resected HCC tissues; however, no further studies verified this result. Therefore, the aim of the present study was to investigate the potential prognostic value of CFHL1 in HCC. First, immunohistochemistry (IHC) and western blot analysis were used to evaluate CFHL1 protein levels in HCC and paired peritumoral tissues. Subsequently, the association between CFHL1 expression and overall survival (OS), pathological characteristics of HCC and time-to-recurrence (TTR) was analyzed and the prognostic value of CFHL1 for postoperative patients with HCC was determined via receiver operating characteristic (ROC) curves. 


\section{Materials and methods}

Patients and specimens. Eight pairs of fresh frozen tissue samples selected for western blotting (collected in January 2017) and a total of 354 formalin-fixed, paraffin-embedded (FFPE) pathological specimens (collected between December 2005 and December 2008) were obtained at the Eastern Hepatobiliary Surgery Hospital (Shanghai, China). The inclusion criteria were as follows: i) No preoperative anticancer treatment; ii) no extrahepatic metastases prior to surgery; and iii) pathological diagnosis of HCC based on the histological diagnostic criteria of the World Health Organization (10). The present study was approved by the Institutional Review Board of the Eastern Hepatobiliary Surgery Hospital and each patient provided written informed consent. FFPE specimens were divided into two groups, namely 76 specimens with paired peritumoral liver tissues and 278 specimens with prognostic data. All 278 cases with prognostic data were followed up after surgery every 3 months for the first year and every 6 months thereafter until December 2013. Serum $\alpha$-fetoprotein (AFP) examination, abdominal ultrasonography and chest $\mathrm{X}$-ray were performed monthly within the first year after surgery and every 3-6 months thereafter. Computed tomography scanning (CT) or magnetic resonance imaging (MRI) of the abdomen was performed every 6 months or immediately upon suspicion of recurrence. The criteria for recurrence were the same as the preoperative diagnostic criteria (11). TTR was defined as the time between tumor resection and tumor recurrence, and OS was defined as the time between surgery and the date of the last follow-up or the date of mortality from any cause. Each FFPE tissue specimen was stained by hematoxylin and eosin and two experienced liver pathologists reviewed the stained sections. Sections (4- $\mu \mathrm{m}$ thick) were placed on slides coated with 3-aminopropyltriethoxysilane. Subsequently, samples were deparaffinized, washed twice with xylene (each $10 \mathrm{~min}$ ) and rehydrated twice with $100 \%$ alcohol for 5 min each, once with 95\% alcohol for $2 \mathrm{~min}$ and once with 70\% alcohol for $2 \mathrm{~min}$. Samples were washed with distilled water and stained with Harris hematoxylin solution for $6 \mathrm{~min}$. Sections were washed under tap water for $5 \mathrm{~min}$, differentiated in $1 \%$ acid alcohol for $10 \mathrm{sec}$, washed under tap water for $1 \mathrm{~min}$ and counterstained with eosin-phloxine solution for $30 \mathrm{sec}$. Subsequently, samples were dehydrated with 95 and $100 \%$ alcohol (each, $5 \mathrm{~min}$ ), and washed twice with xylene (each, 5 min). Xylene based Neutral balsam was used to mount samples. All staining steps were performed at room temperature.

Immunohistochemistry, scoring and tissue microarray (TMA). The method described by Kononen et al (12) was used for the TMAs. Briefly, two experienced pathologists reviewed HE-stained sections and then pre-marked the representative cores in the paraffin blocks. A 1.5-mm diameter tissue cylinder, which was subsequently incorporated into a recipient paraffin block, was punched from a marked area of each block. Subsequently, 4-mm sections were placed on slides coated with 3-aminopropyltriethoxysilane. The paraffin sections were deparaffinized in xylene and rehydrated through decreasing concentrations of ethanol (100, 95 and 85\%; 5 min each). Antigens were retrieved by microwave irradiation for $5 \mathrm{~min}$ in citrate buffer $(\mathrm{pH}$ 6.0) and cooled at room temperature for $120 \mathrm{~min}$, according to the
Table I. Characteristics of patients the samples for western blotting were collected from.

\begin{tabular}{lc}
\hline Variable & Number of patients \\
\hline Sex & \\
Male & 6 \\
Female & 2 \\
Age (years) & \\
$\leq 50$ & 4 \\
$>50$ & 4 \\
HBsAg & \\
Negative & 1 \\
Positive & 7 \\
Serum AFP & \\
$\leq 20$ ng/ml & \\
$>20$ ng/ml & 5 \\
Liver cirrhosis & 3 \\
No & \\
Yes & 5 \\
TNM & 3 \\
I & \\
II & \\
III-IV & 3 \\
Child-pugh score & 2 \\
A & 1 \\
B & \\
Tumor size & \\
$\leq 5$ cm & \\
$>5$ cm & \\
Tumor number & \\
Single & \\
Multiple & \\
Tumor differentiation & \\
Well & \\
Moderate & \\
Poor & \\
Vascular invasion & \\
No & \\
Yes & 5 \\
\hline
\end{tabular}

protocol reported by Jin et al (13) with minor modifications (microwave irradiation was applied for $5 \mathrm{~min}$ in the current study, compared with $3 \mathrm{~min}$ in the previous study). The slides were incubated in $3 \% \mathrm{H}_{2} \mathrm{O}_{2}$ /phosphate-buffered saline to block endogenous peroxidase activity and non-specific binding sites were blocked with goat serum. Samples were treated with Rabbit polyclonal primary antibodies specific to CFHL1 (cat. no. ab103162; 1:50 dilution; cytoplasmic staining; Abcam, Cambridge, MA, USA;) at $4^{\circ} \mathrm{C}$ overnight. Tissue antigens were visualized with an EnVision Detection kit (cat. no. GK500705: Gene Tech Co., Ltd., Hong Kong, China), which included the ChemMate ${ }^{\mathrm{TM}}$ EnVision $^{\mathrm{TM}} / \mathrm{HRP}$ and Rabbit/Mouse (ENV) reagent (a peroxidase-conjugated polymer). Counterstaining with hematoxylin was performed 


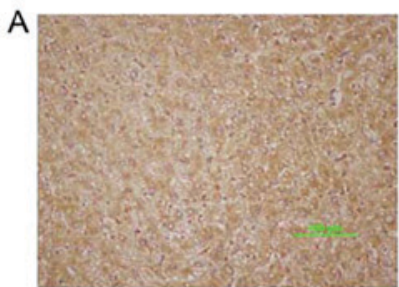

Peritumoral tissue
B

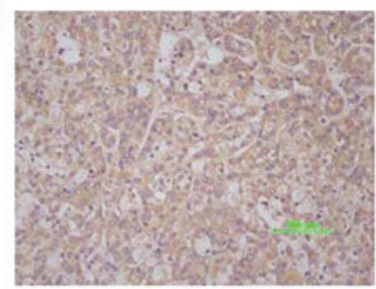

HCC tissue

E

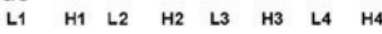

E

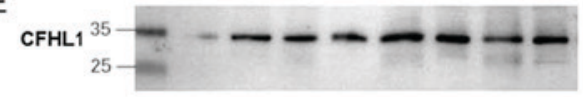

c

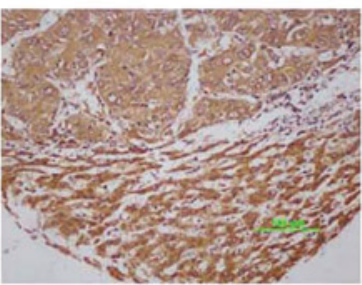

Peritumoral and $\mathrm{HCC}$ tissue

$P=0.8828$
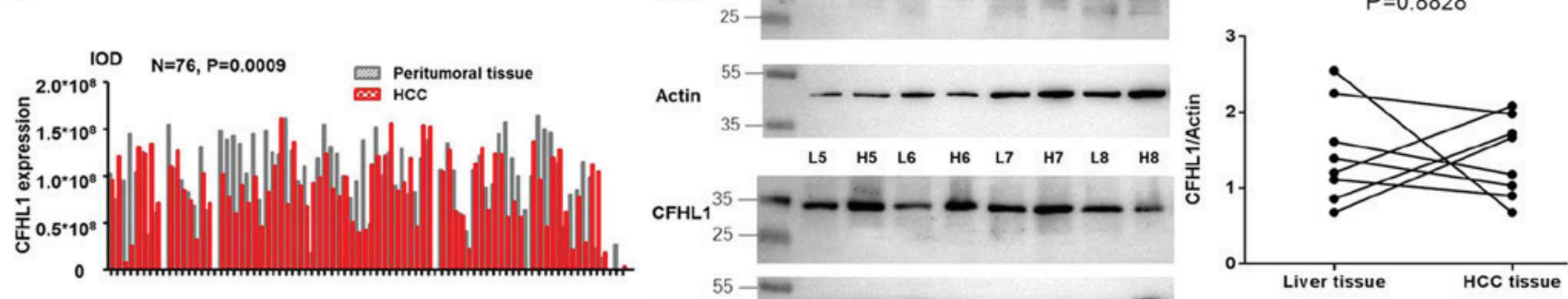
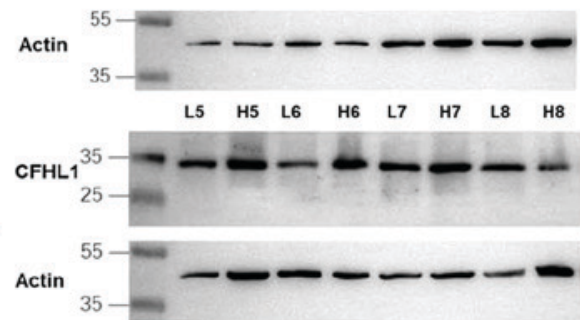

Liver tissue

HCC tissue

Figure 1. Expression level of CFHL1 in HCC and paired peritumoral liver tissues. Expression of CFHL1 in (A) HCC tissue (magnification, x200) and (B) paired peritumoral liver tissue (magnification, x200). (C) HCC and paired peritumoral liver tissue (magnification, x200). (D) IOD for CFHL1 for each case of paired peritumoral liver and HCC tissues from 76 patients. (E) CFHL1 expression was detected by western blot analysis in 8 paired HCC tissues and peritumoral tissue. Western blot analysis of CFHL1 expression in HCC tissue compared with that in paired peritumoral liver tissues. CFHL1, complement factor H-related protein 1; HCC, hepatocellular carcinoma; L, healthy liver tissue; H, HCC tissue; IOD, integrated optical density.

for 5 min. Negative control slides were created for all assays by omission of the primary antibodies. As previously reported (14), the integrated optical density (IOD) of CFHL1 was considered to reflect the expression level. A Leica CCD camera DFC420 connected to a Leica DM IRE2 microscope (Leica Microsystems, Ltd., Milton Keynes, UK) was used for imaging. High-power magnification (x200) with Leica QWin Plus software (version 3; Leica Microsystems, Ltd.) was used to capture images of representative fields. The IOD of each image was counted and measured using Image-Pro Plus software (version 6.0; Media Cybernetics, Inc., Bethesda, MD, USA).

Western blot analysis. Another eight pairs of fresh frozen tissue samples were selected for western blotting which was performed as previously described (15). Characteristics of patients the samples were collected from are summarized in Table I. Briefly, tissue samples were homogenized in a radioimmunoprecipitation assay buffer (Qiagen China Co., Ltd., Shanghai, China) with a cocktail of proteinase inhibitors (Roche Applied Science, Basel, Switzerland) and a cocktail of phosphatase inhibitors (Roche Applied Science). The protein concentrations were determined using the bicinchoninic acid kit (Pierce; Thermo Fisher Scientific Inc., Waltham, MA, USA). Total protein $(20 \mu \mathrm{g} /$ lane) was separated by $10 \%$ SDS-PAGE and transferred to nitrocellulose membranes (Bio-Rad Laboratories, Inc., Hercules, CA, USA). The membranes were incubated with primary antibodies against CFHL1 (1:500 dilution; cat. no. ab103162; Abcam) overnight at $4^{\circ} \mathrm{C}$ and subsequently incubated with Goat anti rabbit IgG-HRP (1:3,000; cat. no. Sc-2004; Santa Cruz Biotechnology, Inc., Dallas, TX, USA) for $60 \mathrm{~min}$ at room temperature. In addition, for detection of loading control protein, anti-actin-HRP conjugated antibody was incubated for $60 \mathrm{~min}$ at room temperature (1:4,000; cat. no. HRP-60008; Proteintech Group, Inc.). After washing the membrane, CFHL1 or actin were visualized using a Pierce ECL Western Blotting Substrate (cat. no. 32106; Pierce; Thermo Fisher Scientific, Inc.) Enhanced Chemiluminescence development solution. The visualized bands were quantified using Quantity One software (Bio-Rad Laboratories, Inc.).

Validation of the association between CFHL1 mRNA expression levels and prognosis. To validate the association between CFHL1 mRNA expression and the prognosis of patients with HCC, the current study analyzed data from the Human Protein Atlas dataset (HPA, www.proteinatlas.org). According to the expected number of fragments per kilobase of transcript sequence per millions base pairs sequenced (FPKM) of CFHL1, patients included in the HPA were classified into low expression CFHL1 and high CFHL1 expression groups. CFHL1 with a median expression FPKM $<1$ were excluded. Kaplan-Meier survival and log-rank analysis were used in the data from HPA website. Genes with $\log$ rank $\mathrm{P}<0.001$ were considered to be prognostic genes. (http://www.proteinatlas. org/ENSG00000244414-CFHR1/pathology/tissue/liver+cancer).

Statistical analysis. Optimization of the cut-point values was based on outcome and the assessment of CFHL1 expression (expressed as IOD) based on X-tile plots (16). Paired t-test was used to analyze mean differences between two sets of observations. A standard log-rank method was used to analyze the cut-off scores, which were derived from 278 cases. The association between CFHL1 expression and patient survival was analyzed via the Mantel Cox log-rank test and Kaplan-Meier analysis. A $\chi^{2}$ test was used to analyze the association between clinicopathological characteristics and CFHL1 expression. The SPSS statistical software package (version 13.0; SPSS, Inc., Chicago, IL, USA) was used for data analysis. $\mathrm{P}<0.05$ was considered to indicate a statistically significant difference. 


\section{Results}

CFHL1 is downregulated in HCC tissues at the protein level. Cytoplasmic expression of CFHL1 was detected in both HCC and peritumoral tissues (Fig. 1A-C). The results demonstrated that CFHL1 expression was downregulated in 51 and upregulated in $25 \mathrm{HCC}$ tissue samples, compared with the peritumoral tissue samples ( $\mathrm{P}=0.0009$; Fig. 1D). Additionally, the expression level of CFHL1 was analyzed in 8 paired HCC and peritumoral tissue samples by western blotting. The expression level of CHFL1 was lower in HCC tissues compared with peritumoral samples; however, the difference was not statistically significant (Fig. 1).

Association between clinicopathological characteristics and CFHL1 expression. In order to determine the association between CFHL1 expression and clinicopathologcal variables in patients with $\mathrm{HCC}$, data from the 354 patients with $\mathrm{HCC}$ were collected and evaluated by $\chi^{2}$ test. The results demonstrated that low CFHL1 expression was associated with vascular invasion $(\mathrm{P}=0.024)$ and serum AFP levels $(\mathrm{P}=0.039)$; however, no significant associations were observed with other clinicopathological parameters, including liver cirrhosis, sex, TNM stage, age, HBsAg, tumor size, Child-Pugh class, tumor differentiation or tumor number (all $\mathrm{P}>0.05$; Table II).

Association of low CFHL1 expression with OS and TTR in patients with HCC. The association of low CFHL1 expression with OS and TTR was further assessed in 278 cases of HCC via univariate and multivariate analyses (Table III). The results demonstrated that tumor size, liver cirrhosis, TNM stage, tumor number, CFHL1 expression and vascular invasion were significant prognostic factors for OS and TTR on univariate analysis (all $\mathrm{P}<0.05)$. With regard to tumor differentiation in the univariate analysis, there was a statistically significant difference for TTR $(\mathrm{P}=0.039)$, while there was no difference for $\mathrm{OS}(\mathrm{P}=0.271)$. In the multivariate analysis, liver cirrhosis, tumor size, low CFHL1 expression and tumor number were independent prognostic factors for OS and TTR (all $\mathrm{P}<0.05$ ). Furthermore, as presented in Fig. 2, both OS and TTR were significantly worse in the low CFHL1 expression group (number of patients, 207) compared with those in the high CFHL1 expression group $(n=71$; all $\mathrm{P}<0.05)$.

Further validation in TCGA and The Human Protein Atlas datasets. To validate the expression of CFHL1, CFHL1 mRNA expression data in 50 pairs of HCC and normal tissue samples were obtained from the database of The Cancer Genome Atlas (TCGA; cancergenome.nih.gov). Fig. 3A revealed that CFHL1 mRNA expression levels in 50 pairs of $\mathrm{HCC}$ were statistically lower compared with normal tissue samples $(\mathrm{P}=0.0195)$. CFHL1 mRNA expression level of 365 patients in The Human Protein Atlas dataset (www.proteinatlas.org) was used for prognosis validation. Results revealed that low CFHL1 mRNA level was associated with worse prognosis compared with the high-expression group ( $\mathrm{P}=8.71 \times 10^{-6}$; Fig. 3B).

Prognostic model including tumor size, tumor number, liver cirrhosis and CFHL1. Based on the results presented in Table III, a new prognostic model was proposed that included liver cirrhosis, tumor size, tumor number and CFHL1
Table II. CFHL1 expression level associations with pathological characteristics of HCC.

\begin{tabular}{|c|c|c|c|}
\hline \multirow[b]{2}{*}{ Variable } & \multicolumn{2}{|c|}{ CFHL1 } & \multirow[b]{2}{*}{ P-value } \\
\hline & Low & High & \\
\hline Sex & & & 0.554 \\
\hline Male & 233 & 78 & \\
\hline Female & 34 & 9 & \\
\hline Age (years) & & & 0.426 \\
\hline$\leq 51$ & 142 & 42 & \\
\hline$>51$ & 125 & 45 & \\
\hline $\mathrm{HBsAg}^{\mathrm{a}}$ & & & 0.196 \\
\hline Negative & 50 & 11 & \\
\hline Positive & 215 & 75 & \\
\hline Serum AFPa & & & 0.039 \\
\hline$\leq 20 \mathrm{ng} / \mathrm{ml}$ & 88 & 39 & \\
\hline $20 \mathrm{ng} / \mathrm{ml}$ & 178 & 47 & \\
\hline Liver cirrhosis & & & 0.463 \\
\hline No & 72 & 27 & \\
\hline Yes & 195 & 60 & \\
\hline TNM & & & 0.182 \\
\hline $\mathrm{I}$ & 79 & 35 & \\
\hline II & 142 & 3 & \\
\hline III-IV & 46 & 13 & \\
\hline Child-pugh score ${ }^{a}$ & & & 0.953 \\
\hline A & 243 & 78 & \\
\hline $\mathrm{B}$ & 24 & 8 & \\
\hline Tumor size & & & 0.260 \\
\hline$\leq 5 \mathrm{~cm}$ & 78 & 20 & \\
\hline$>5 \mathrm{~cm}$ & 189 & 67 & \\
\hline Tumor number & & & 0.324 \\
\hline Single & 210 & 64 & \\
\hline Multiple & 57 & 23 & \\
\hline Tumor differentiation & & & 0.881 \\
\hline Well & 22 & 8 & \\
\hline Moderate & 226 & 74 & \\
\hline Poor & 19 & 5 & \\
\hline Vascular invasion & & & 0.024 \\
\hline No & 90 & 41 & \\
\hline Yes & 177 & 46 & \\
\hline
\end{tabular}

${ }^{a}$ Based on HBsAg, the data of three patients are missing; Based on AFP, the data of two patients are missing; Based on Child-Pugh score, the data of one patient is missing. CFHL1, Complement factor $\mathrm{H}$-related protein 1 .

for both OS and TTR. As presented in Fig. 4, the ROC curve analysis demonstrated that the predictive accuracy of the new prognostic model [area under the curve (AUC) 0.697 for OS and 0.702 for TTR] was higher compared with that for any other factor and the prognostic model without CFHL1 (liver cirrhosis/tumor number/tumor size combination). The AUC 
Table III. Univariate and multivariate analyses of clinico-pathological factors associated with OS and TTR.

\begin{tabular}{|c|c|c|c|c|c|c|c|c|}
\hline \multirow[b]{3}{*}{ Factor } & \multicolumn{4}{|c|}{ OS } & \multicolumn{4}{|c|}{ TTR } \\
\hline & \multirow{2}{*}{$\begin{array}{l}\text { Univariate } \\
\text { P-value }\end{array}$} & \multicolumn{3}{|c|}{ Multivariate } & \multirow{2}{*}{$\begin{array}{l}\text { Univariate } \\
\text { P-value }\end{array}$} & \multicolumn{3}{|c|}{ Multivariate } \\
\hline & & HR & $95 \% \mathrm{Cl}$ & P-value & & HR & $95 \% \mathrm{Cl}$ & P-value \\
\hline Sex, male vs. female & 0.637 & & & & 0.946 & & & \\
\hline Age,$\leq 51$ vs. $>51$ & 0.678 & & & & 0.395 & & & \\
\hline HBsAg, positive vs. negative & 0.174 & & & & 0.173 & & & \\
\hline $\operatorname{Serum} \operatorname{AFP}(\mathrm{ng} / \mathrm{ml}), \leq 20$ vs. $>20$ & 0.162 & & & & 0.248 & & & \\
\hline Liver cirrhosis, yes vs. no & 0.005 & 1.933 & $1.283-2.911$ & 0.002 & $<0.0001$ & 2.176 & $1.476-2.913$ & $<0.0001$ \\
\hline TNM, I vs. II vs. III-IV & $<0.0001$ & & & & $<0.0001$ & & & \\
\hline Child-pugh score, A vs. B & 0.379 & & & & 0.126 & & & \\
\hline Tumor size,$\leq 5$ vs. $>5$ & 0.025 & 1.736 & $1.104-2.731$ & 0.017 & 0.006 & 1.937 & $1.307-2.871$ & 0.001 \\
\hline Tumor number, single vs. multiple & $<0.0001$ & 2.142 & $1.465-3.132$ & $<0.0001$ & $<0.0001$ & 2.082 & $1.480-2.929$ & $<0.0001$ \\
\hline $\begin{array}{l}\text { Tumor differentiation, } \\
\text { well vs. moderate vs. poor }\end{array}$ & 0.271 & & & & 0.039 & & & \\
\hline Vascular invasion, no vs. yes & 0.015 & & & & 0.025 & & & \\
\hline CFHL1, low vs. high & 0.003 & 0.470 & $0.294-0.751$ & 0.002 & 0.020 & 0.630 & $0.431-0.920$ & 0.017 \\
\hline
\end{tabular}

A

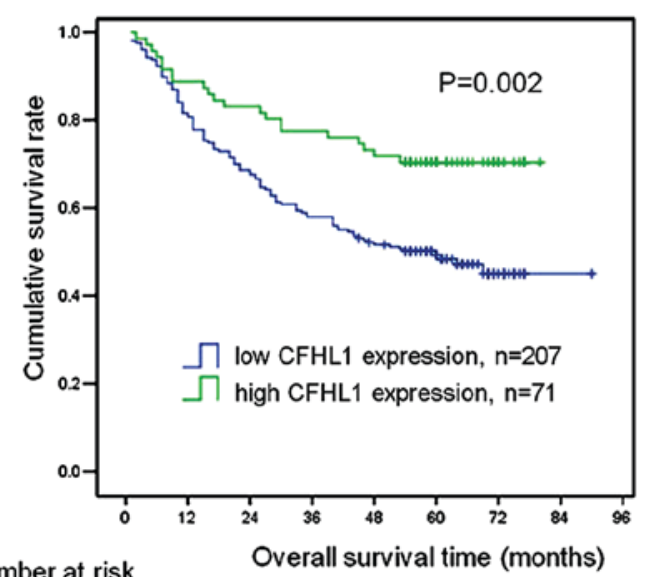

Number at risk

$\begin{array}{lllllllll}\text { Low CFHL1 } & 207 & 169 & 142 & 120 & 106 & 60 & 13 & 1\end{array}$

$\begin{array}{llllllll}\text { High CFHL1 } & 71 & 63 & 59 & 55 & 52 & 32 & 10\end{array}$

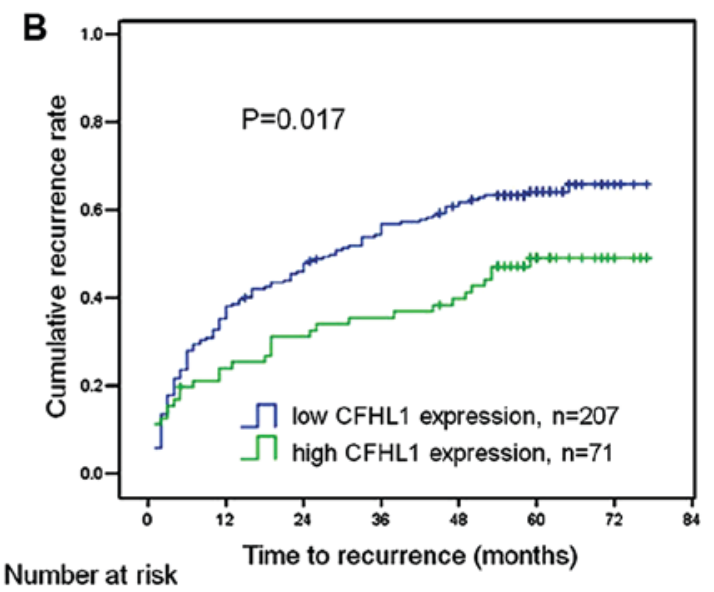

$\begin{array}{lllllllll}\text { Low CFHL1 } & 207 & 134 & 111 & 92 & 77 & 40 & 6\end{array}$

$\begin{array}{llllllll}\text { High CFHL1 } & 71 & 53 & 48 & 45 & 41 & 23 & 7\end{array}$

Figure 2. Kaplan-Meier curves for OS and TTR in HCC patients (n=278). (A) OS. (B) TTR. CFHL1, complement factor H-related protein 1; HCC, hepatocellular carcinoma; OS, overall survival; TTR, time-to-recurrence.
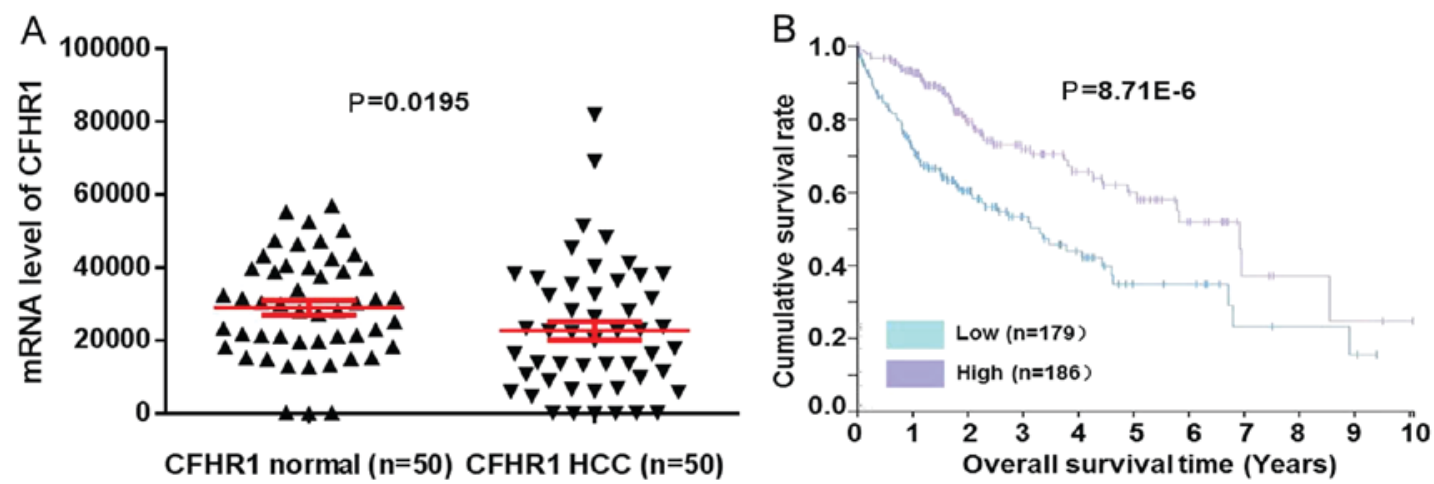

Figure 3. Validation of CFHL1 mRNA expression and prognosis in TCGA and human protein atlas datasets. (A) CFHL1 mRNA expression level in 50 pairs of HCC were statistically lower than that in normal tissue samples ( $\mathrm{P}=0.0195$, data from TCGA). (B) Low CFHL1 mRNA level had worse prognosis ( $\mathrm{P}=8.71 \mathrm{E}-6$, data from human protein atalas). CFHL1, complement factor H-related protein 1; HCC, hepatocellular carcinoma; CFHR1, alternate name of CFHL1. 

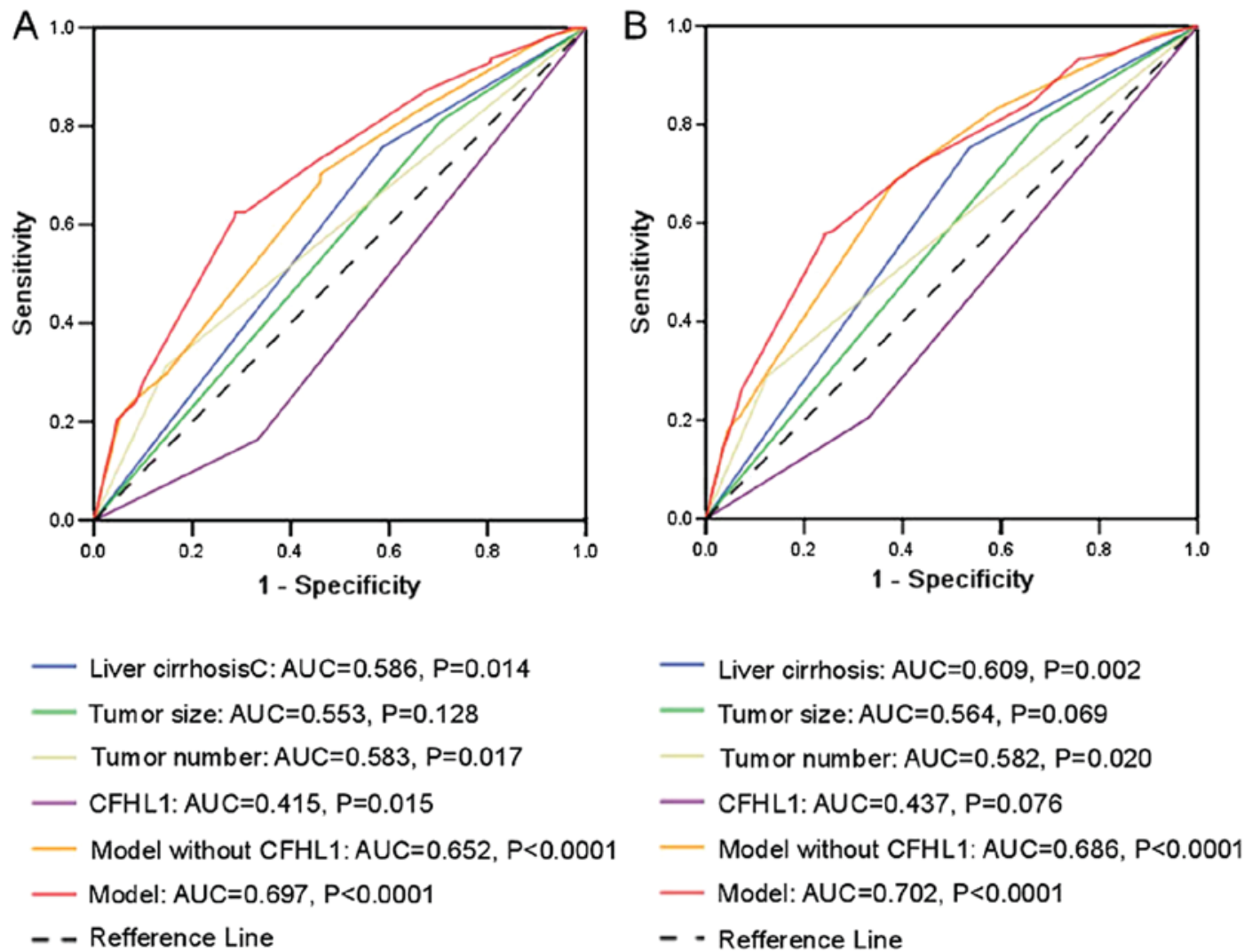

Figure 4. ROC curves and AUC of the novel prognostic model for (A) OS and (B) TTR. AUC demonstrated that the accuracy of the prognostic model was higher compared to that of liver cirrhosis, tumor size, tumor number and CFHL1 alone, and that of the prognostic model without CFHL1 (liver cirrhosis/tumor size/tumor number combination) for both OS and TTR. CFHL1, complement factor H-related protein 1; OS, overall survival; TTR, time-to-recurrence; ROC, receiver operating characteristic; AUC, area under the ROC curve.

values for OS were $0.553,0.586,0.583,0.652$ and 0.415 for tumor size, liver cirrhosis, tumor number, model without CFHL1 and CFHL1, respectively, whereas the respective AUC values for TTR were $0.564,0.609,0.582,0.686$ and 0.437 .

\section{Discussion}

The present study demonstrated that CFHL1 expression was decreased in HCC tissues, and patients with low CFHL1 expression exhibited worse OS and TTR. Furthermore, after proposing a new prognostic model, the AUC of this new model was higher compared with that of the liver cirrhosis/tumor size/tumor number combination. Collectively, these data indicated that decreased CFHL1 expression was associated with poor outcome in patients with HCC.

To the best of our knowledge, there are no studies in the literature on the association between CFHL1 and cancer, including HCC. In the current study, the expression of CFHL1 was examined in HCC and paired peritumoral tissues by western blot analysis and immunohistochemical examination. The results revealed that CFHL1 was downregulated in HCC tissues, with a significant difference between HCC and peritumoral tissues, indicating that CFHL1 may be a risk factor for HCC. In addition, based on the Kaplan-Meier analysis, an association was observed between CFHL1 protein expression and clinical outcome in patients with HCC after surgery. Patients with low CFHL1 expression had worse OS and shorter TTR compared with cases with high CFHL1 expression. The multivariate analysis indicated that CFHL1 was an independent postoperative predictor of recurrence and OS. Furthermore, in order to validate the prognostic value of decreased CFHL1 expression in postoperative patients with HCC, ROC curve analysis and bioinformatics analysis were performed. The results revealed that the new prognostic model was superior to that without CFHL1 (liver cirrhosis/tumor size/tumor number combination) and low CFHL1 mRNA level had worse prognosis, indicating that CFHL1 may be a reliable prognostic factor for postoperative patients with HCC.

To the best of our knowledge, the present study was the first to investigate the association between CFHL1 expression and the prognosis of postoperative patients with $\mathrm{HCC}$, however, there were several limitations. First, similar studies on the role of CFHL1 in other types of cancer, which could expand the prognostic value of CFHL1, are required. Second, more in vivo and in vitro studies should be performed in the future to elucidate why the downregulated expression of CFHL1 is associated with unfavorable prognosis in postoperative HCC patients. Previous studies have demonstrated an association of CFHL1 with human neutrophil granulocytes (17) and the human immune system (18).

In conclusion, the current study was the first to demonstrate that CFHL1 may be of high prognostic value in postoperative patients with $\mathrm{HCC}$ and it may be used as a new prognostic biomarker in such individuals. However, further studies, particularly on the association between CFHL1 and the tumor microenvironment, should be conducted in the future. 


\section{Acknowledgements}

Not applicable.

\section{Funding}

The present study was supported by the youth projects of the general logistics department of Chinese people's liberation army (grant no. 13QNP101).

\section{Availability of data and materials}

The datasets used and/or analyzed during the current study are available from the corresponding author on reasonable request.

\section{Authors' contributions}

XYY and YH were responsible for the study conception and design. Experiments were performed by HF and FF. HF, FF, LY and MX were responsible for patient follow-up. HF and FF performed data analysis. $\mathrm{HF}$ and $\mathrm{FF}$ were responsible for the drafting of the manuscript. XYY and YH performed the review and editing of the manuscript. All authors have read and approved the final version of this manuscript.

\section{Ethics approval and consent to participate}

The study protocol was approved by the Ethics Committee of the Eastern Hepatobiliary Surgery Hospital and all the patients signed written informed consent.

\section{Patient consent for publication}

Not applicable.

\section{Competing interests}

The authors declare that they have no competing interests.

\section{References}

1. Li F, Guo Z and Wang H: Influencing elements and treatment strategies associated with the relapse of hepatocellular carcinoma after surgery. Hepatogastroenterology 60: 1148-1155, 2013.

2. Bruix J and Sherman M; Practice Guidelines Committee, American Association for the Study of Liver Diseases: Management of hepatocellular carcinoma. Hepatology 42: 1208-1236, 2005.

3. Umeda S, Kanda M and Kodera Y: Emerging evidence of molecular biomarkers in hepatocellular carcinoma. Histol Histopathol 33: 343-355, 2018.

4. Skerka C, Timmann C, Horstmann RD and Zipfel PF: Two additional human serum proteins structurally related to complement factor $\mathrm{H}$. Evidence for a family of factor $\mathrm{H}$-related genes. J Immunol 148: 3313-3318, 1992.
5. Siegel C, Hallström T, Skerka C, Eberhardt H, Uzonyi B Beckhaus T, Karas M, Wallich R, Stevenson B, Zipfel PF and Kraiczy P: Complement factor H-related proteins CFHR2 and CFHR5 represent novel ligands for the infection-associated CRASP proteins of Borrelia burgdorferi. PLoS One 5: e13519, 2010.

6. Kinders R, Jones T, Root R, Bruce C, Murchison H, Corey M, Williams L, Enfield D and Hass GM: Complement factor $\mathrm{H}$ or a related protein is a marker for transitional cell cancer of the bladder. Clin Cancer Res 4: 2511-2520, 1998.

7. Heicappell R, Wettig IC, Schostak M, Müller M, Steiner U, Sauter T and Miller K: Quantitative detection of human complement factor $\mathrm{H}$-related protein in transitional cell carcinoma of the urinary bladder. Eur Urol 35: 81-87, 1999.

8. Malkowicz SB: The application of human complement factor H-related protein (BTA TRAK) in monitoring patients with bladder cancer. Urol Clin North Am 27: 63-73, ix, 2000.

9. Yang JD, Seol SY, Leem SH, Kim YH, Sun Z, Lee JS, Thorgeirsson SS, Chu IS, Roberts LR and Kang KJ: Genes associated with recurrence of hepatocellular carcinoma: Integrated analysis by gene expression and methylation profiling. J Korean Med Sci 26: 1428-1438, 2011.

10. Jin GZ, Yu WL, Dong H, Zhou WP, Gu YJ, Yu H, Yu H, Lu XY, Xian ZH, Liu YK, et al: SUOX is a promising diagnostic and prognostic biomarker for hepatocellular carcinoma. J Hepatol 59: 510-517, 2013.

11. Tan N, Liu Q, Liu X, Gong Z, Zeng Y, Pan G, Xu Q and He S: Low expression of B-cell-associated protein 31 in human primary hepatocellular carcinoma correlates with poor prognosis. Histopathology 68: 221-229, 2016.

12. Kononen J, Bubendorf L, Kallioniemi A, Bärlund M, Schraml P, Leighton S, Torhorst J, Mihatsch MJ, Sauter G and Kallioniemi OP: Tissue microarrays for high-throughput molecular profiling of tumor specimens. Nat Med 4: 844-847, 1998.

13. Jin GZ, Li Y, Cong WM, Yu H, Dong H, Shu H, Liu XH, Yan GQ, Zhang L, Zhang Y, et al: iTRAQ-2DLC-ESI-MS/MS based identification of a new set of immunohistochemical biomarkers for classification of dysplastic nodules and small hepatocellular carcinoma. J Proteome Res 10: 3418-3428, 2011.

14. Zhu XD, Zhang JB, Zhuang PY, Zhu HG, Zhang W, Xiong YQ, Wu WZ, Wang L, Tang ZY and Sun HC: High expression of macrophage colony-stimulating factor in peritumoral liver tissue is associated with poor survival after curative resection of hepatocellular carcinoma. J Clin Oncol 26: 2707-2716, 2008.

15. Jin H, Wang C, Jin G, Ruan H, Gu D, Wei L, Wang H, Wang N, Arunachalam E, Zhang Y, et al: Regulator of calcineurin 1 gene isoform 4, down-regulated in hepatocellular carcinoma, prevents proliferation, migration, and invasive activity of cancer cells and metastasis of orthotopic tumors by inhibiting nuclear translocation of NFAT1. Gastroenterology 153: 799-811.e33, 2017.

16. Camp RL, Dolled-Filhart $M$ and Rimm DL: X-tile: A new bio-informatics tool for biomarker assessment and outcome-based cut-point optimization. Clin Cancer Res 10: 7252-7259, 2004.

17. Losse J, Zipfel PF and Józsi M: Factor H and factor H-related protein 1 bind to human neutrophils via complement receptor 3 , mediate attachment to Candida albicans, and enhance neutrophil antimicrobial activity. J Immunol 184: 912-921, 2010.

18. Siegel C, Schreiber J, Haupt K, Skerka C, Brade V, Simon MM, Stevenson B, Wallich R, Zipfel PF and Kraiczy P: Deciphering the ligand-binding sites in the Borrelia burgdorferi complement regulator-acquiring surface protein 2 required for interactions with the human immune regulators factor $\mathrm{H}$ and factor $\mathrm{H}$-like protein 1. J Biol Chem 283: 34855-34863, 2008. 